\title{
Querelles de frontière à la gare de Paris-Austerlitz : la rue Watt et les ponts du Paris-Orléans, 1839-1937
}

Border conflicts at Paris-Austerlitz station: rue Watt and the Paris-Orléans railway bridges, $1839-1937^{*}$

\section{Karen Bowie}

\section{OpenEdition}

\section{Journals}

Édition électronique

URL : https://journals.openedition.org/rhcf/445

DOI : 10.4000/rhcf.445

Éditeur

Rails \& histoire

Édition imprimée

Date de publication : 31 mai 2008

Pagination : 127-157

ISSN : 0996-9403

\section{Référence électronique}

Karen Bowie, «Querelles de frontière à la gare de Paris-Austerlitz : la rue Watt et les ponts du Paris-

Orléans, 1839-1937 », Revue d'histoire des chemins de fer [En ligne], 38 | 2008, mis en ligne le 10 mai

2011, consulté le 22 avril 2022. URL : http://journals.openedition.org/rhcf/445 ; DOI : https://doi.org/ $10.4000 /$ rhcf. 445 


\title{
Querelles de frontière à la gare de Paris-Austerlitz : la rue Watt et les ponts du Paris-Orléans, 1839-1937
}

\author{
Karen BOWIE
}

ituée à Paris dans le XIII ${ }^{\mathrm{e}}$ arrondissement, entre la Bibliothè-
que nationale de France et le boulevard périphérique, la rue
Watt relie les quais de la Seine et la rue du Chevaleret. Elle est perpendiculaire à l'axe des voies ferrées de la gare d'Austerlitz qui la traversent sur des ponts. Depuis les origines de la gare, elle constitue un point sensible de limite entre le territoire ferroviaire et l'espace public de la ville (fig. 1).

Figure 1.

Le XIII ${ }^{\mathrm{e}}$ arrondissement dans les années 1960, avant ses transformations récentes. On peut y apercevoir entre autres l'ancienne gare aux marchandises SNCF, dont le site est aujourd'hui occupé par la Bibliothèque Nationale de France. La rue Watt relie le quai de la Gare et la rue du Chevaleret, entre la rue de Tolbiac et le boulevard Masséna.

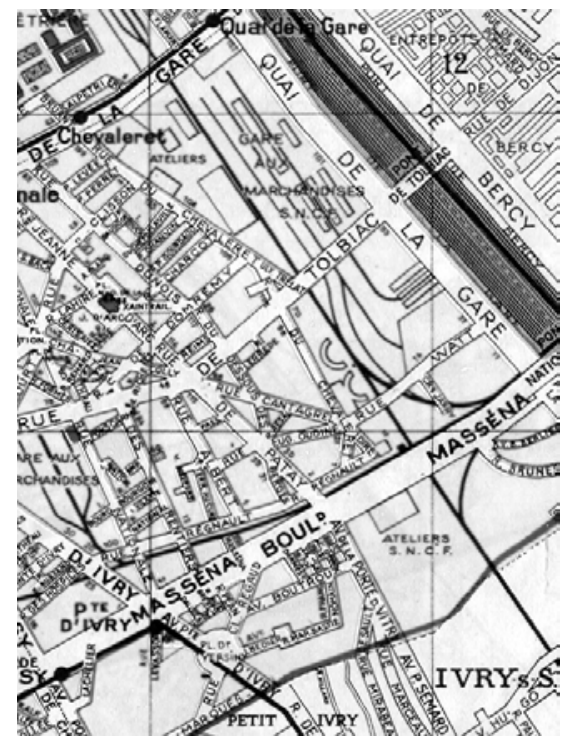


Les ponts ferroviaires qui la traversent constituent un ouvrage complexe, qui portait au XIX ${ }^{\mathrm{e}}$ siècle non seulement les voies des grandes lignes Paris-Orléans, mais aussi, à partir des années 1850, les voies de raccordement entre la gare d'Austerlitz et le chemin de fer de Ceinture. Pendant les années 1870, on envisagera de remplacer ce raccordement alors intra-muros par une voie dite extra-muros, à l'extérieur des murs de fortification. La mise en œuvre de ce projet qui comportait des modifications importantes des ponts de la rue Watt fit l'objet de négociations qui durèrent plusieurs décennies.

\section{Le cadre de réalisation de la présente étude}

Issu d'un rapport commandité par la SEMAPA (Société d'économie mixte d'aménagement de Paris), le travail que nous présentons ici doit son existence à un contexte particulier. La rue Watt avait attiré l'attention de certaines associations de défense du patrimoine pendant les années 1990, alors que de grandes opérations d'aménagement étaient en cours, dans le cadre de la zone d'aménagement concertée Paris Rive Gauche, sur des terrains situés entre la gare d'Austerlitz et le boulevard périphérique. Cette rue se présentait en effet comme un lieu insolite où le chemin de fer passait au-dessus de la voie publique sur des ponts métalliques en treillis qui pouvaient laisser passer une lumière tamisée (fig. 2). Situé près de la «zone » des anciennes fortifications, cet ouvrage avait donné lieu à des représentations en bandes dessinées, chansons populaires et romans policiers qui lui avaient conféré une certaine célébrité. Cependant, l'histoire de sa construction et de son rôle dans l'évolution du site n'avait jamais fait l'objet de travaux historiques ${ }^{1}$.

1- Cela même si même si deux excellentes études sur l'ensemble du site ont été réalisées à la demande de l'aménageur SEMAPA, dont une disponible en librairie : Gilles-Antoine Langlois, De la Salpêtrière à la Bibliothèque nationale de France. Histoire d'un quartier de Paris, Paris, Somogy/Semapa, 2000. Voir aussi Pascale Cros, Virginie Gala et Marianne Thébert, « Mémoire des lieux et identité sociale. Une nouvelle donnée de l'aménagement du territoire, étude d'un cas : ZAC Paris Rive Gauche », DESS d'Aménagement et d'urbanisme, sous la direction de F. Beaucire et G. Burgel, université de Paris X Nanterre, septembre 1998. Si nous présentons ici la première étude historique des ponts de la rue Watt, plusieurs autres éléments marquants du site ont déjà fait l'objet d'études monographiques : pour l'usine d'air comprimé (SUDAC), voir Thierry Poujol, « Le réseau d'air comprimé : une stratégie ambitieuse, mais un destin parisien ", dans François Caron, Jean Derens, et Luc Passion (dir.), Paris et ses réseaux: naissance d'un mode de vie urbain, $\mathrm{XIX}^{\mathrm{e}}$ - XX $\mathrm{XX}^{\mathrm{e}}$ siècles, Paris, Bibliothèque historique de la Ville de Paris, 1990, ainsi que Hélène Jantzen, « L'usine d'air comprimé Sudac » dans Jean-François Belhoste et Paul Smith, Patrimoine Industriel, cinquante sites en France, Paris, Éditions du Patrimoine, 1997. On peut aussi consulter, concernant les Grands Moulins, Le Meunier de Paris, 75 ans d'histoire des Grands Moulins de Paris, Paris, Somogy, 1996 ; voir aussi Karen Bowie, " A note on the "Paris Rive Gauche" Development Project : Opportunities and Challenges for Industrial Heritage ", Patrimoine de l'Industriel / Industrial Patrimony, $\mathbf{n}^{\circ} 10$ (2003), p. 97-102. 
La recherche que nous avons réalisée se voulait ainsi plus historique et documentaire que proprement patrimoniale. En effet, il nous semblait qu'une étude documentaire était un préalable à des débats sur les choix d'aménagement ${ }^{2}$.

L'étude du «cas » des ponts de la rue Watt apporte surtout ainsi des éclairages sur la manière dont la Compagnie du chemin de fer Paris-Orléans menait ses relations avec la municipalité, sur une période allant des débuts de l'implantation ferroviaire sur le site en 1839 jusqu’à la création de la SNCF presqu'un siècle plus tard.

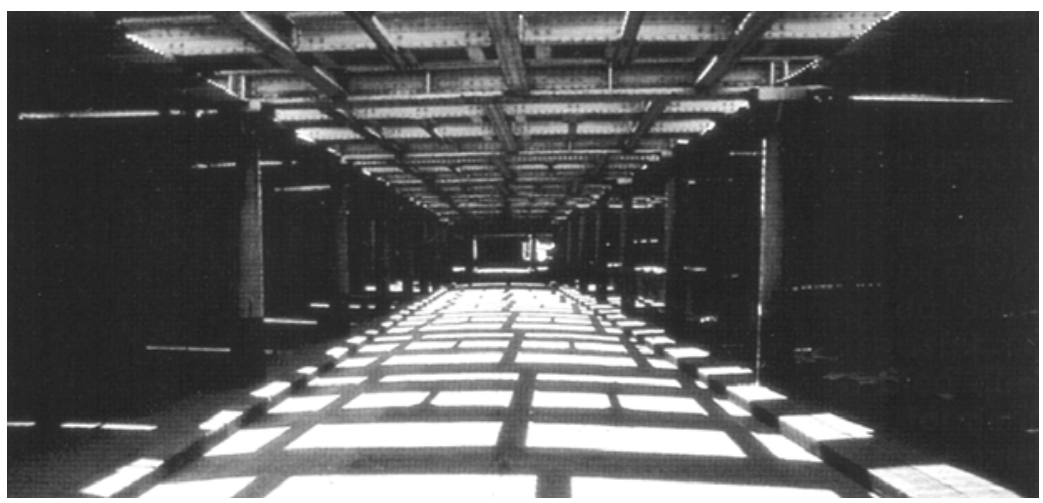

Figure 2. La rue Watt. Photographie publiée dans Paris-Projetn ${ }^{\circ} 29$, septembre 1990. Cl. d.r.

2- Voir Karen Bowie, «Étude historique sur les emprises ferroviaires de la ZAC Paris Rive-Gauche », SEMAPA/IPRAUS, mars 2004. 


\section{Ponts ferroviaires sur la rue Watt. Chronologie sommaire 1839-1937}

1839 : origines du pont. Longueur $8 \mathrm{~m}$; ouverture $3 \mathrm{~m}$. Poutres en bois. 1854 : longueur $24 \mathrm{~m}$; ouverture $5 \mathrm{~m}$. Tablier métallique.

[Années 1850 : mis en place d'un pont de $5 \mathrm{~m}$ d'ouverture sur la rue

Watt, côté Seine, portant les voies de raccordement avec la Petite

Ceinture : voir fig. 5].

1867 : allongement à $43 \mathrm{~m}$ de longueur du tablier métallique de $5 \mathrm{~m}$ d'ouverture.

1873 et 1876 : approbation de projets portant la longueur du pont à $125 \mathrm{~m}$ et son ouverture à $12 \mathrm{~m}$. Ces projets prévoient la suppression du raccordement intra-muros avec le chemin de fer de Ceinture et son remplacement par un raccordement extra-muros (fig. 6).

1881-1883 : réalisation sur une longueur de $71 \mathrm{~m}$ seulement (et non pas 125) du nouveau pont de $12 \mathrm{~m}$ d'ouverture, approuvé en 1873 et 1876 (fig. 3).

«La zone nécessaire au passage de chaque voie est constituée par deux poutres principales à 3 travées solidaires, dont 1 centrale de

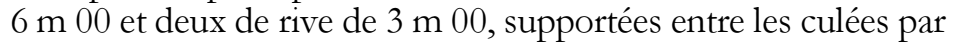
des colonnes en fonte. Les poutres principales sont reliées par des pièces de pont, espacées entre elles de $1 \mathrm{~m} \mathrm{90,} \mathrm{la} \mathrm{voie} \mathrm{repo-}$ sant sur des longrines, supportées par des longerons en forme de caisson ». [Centre des archives historiques de la SNCF, 236LM30/4/5. Chemin de fer d'Orléans. Service de la voie. Dossier prescrit par la Circulaire Ministérielle du 29 août $1891.1^{\circ}$ Historique de l'ouvrage. 28 janvier 1897.]
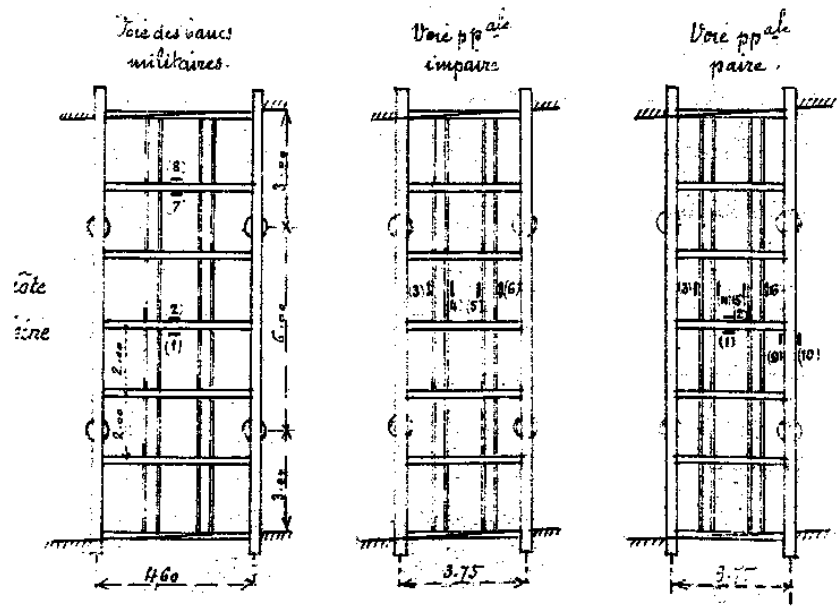

Figure 3. "Pont inférieur de la rue Watt sous les voies de la gare d'Ivry, composé de 3 travées solidaires dont 2 de rive de $3 \mathrm{~m} 00$ et 1 centrale de $6 \mathrm{~m} 00 \mathrm{~d}$ 'ouverture ". Centre des archives historiques de la SNCF, 236LM27/6/3. 
Le nouveau pont de $12 \mathrm{~m}$ porte les voies de la Cie $\mathrm{PO}$, mais le pont de raccordement avec le chemin de fer de Ceinture, de $5 \mathrm{~m}$ d'ouverture seulement, est laissé en place sur le côté Seine de la rue Watt (fig. 5).

[1884 : mise en place d'un platelage en tôle striée dans les entrevoies adjacentes à certaines voies.]

[1887 : mise en place d'un plancher en bois de part et d'autre de certaines voies, « pour empêcher l'huile et l'eau provenant des machines de tomber sur les passants à l'intérieur du pont. »]

1896 : plainte du commissaire-voyer du XIII ${ }^{\mathrm{e}}$ arrondissement concernant les étais en bois du pont de raccordement avec la Ceinture. Projet de remplacement des étais par un mur de soutènement. Projet nonréalisé de reconstruction du pont établi sous le raccordement de ceinture (voir fig. 8).

1905: adjonction d'un tablier de $12 \mathrm{~m}$ d'ouverture côté rue du Chevaleret, à l'occasion du doublement de voies entre Paris et Brétigny et la réalisation d'une nouvelle voie de sortie du dépôt. (Mise en œuvre de poutres métalliques approvisionnées en 18811883.)

1908 : adjonction d'un tablier de 12 m d'ouverture côté rue du Chevaleret suite à la mise en relation d'une nouvelle remise avec une rotonde existante. (Mise en œuvre de poutres métalliques approvisionnées en 1881-1883.)

1913-1914 : remplacement du pont du raccordement et adjonction de 3 tabliers métalliques de $12 \mathrm{~m}$ d'ouverture (côté Seine de la rue Watt).

1917-1923 : efforts de la Cie PO pour obtenir la «suppression pure et simple » de la rue Watt.

1924-1928 : projets pour la déviation de la rue Watt ; projets pour remplacer les ponts ferroviaires par un "pont supérieur» faisant passer la chaussée de la voie publique au-dessus des voies ferrées.

1928 : allongement du pont sur $70 \mathrm{~m}$ côté rue du Chevaleret; poutrelles en acier d'une seule portée (sans colonnes intermédiaires) enrobées dans du béton.

1937 : remplacement de 4 tabliers sous voies principales par des tabliers en acier d'une seule portée. 


\section{Un fonds d'archives exceptionnel, des éclairages nouveaux}

Notre étude se fonde essentiellement sur des documents conservés au Centre des archives historiques de la SNCF, au Mans ${ }^{3}$. Les archives de la gare d'Austerlitz, contrairement à la grande majorité des archives de la Compagnie du Paris-Orléans, ne furent pas versées aux Archives Nationales au moment de la création de la SNCF en 19374. Ces documents, considérés comme pouvant être toujours nécessaires à la gestion du site, furent conservés sur place dans la gare et étaient donc inaccessibles aux chercheurs jusque vers la fin des années 1990.

Cette documentation désormais accessible permet donc d'apporter un éclairage inédit sur le fonctionnement, pendant un siècle, de la Compagnie du Paris-Orléans lorsqu'elle aménage et réaménage l'immense zone occupée par la tête de son réseau, à cheval entre Paris et Ivry. Le "cas» des négociations concernant les ponts ferroviaires et l'aménagement de la rue Watt, bien représenté dans les archives du Mans, nous a ainsi permis de découvrir un aspect fondamental de l'activité de l'entreprise ferroviaire resté jusqu'ici peu abordé par les travaux historiques.

Cette documentation restitue le travail quotidien des ingénieurs responsables de l'aménagement ainsi que leurs modes de communication entre eux. Le plus souvent, cette communication se fait par le moyen de rapports techniques commentés, les discussions et débats étant consignés dans des annotations manuscrites en marge d'un même document transmis et retransmis d'un service à un autre: c'est sur ce type de document que repose l'essentiel de notre étude.

Soulignons cependant que l'archive exploitée pour réaliser notre recherche est à la fois abondante et lacunaire : les aléas de sa conservation ont fait que certaines séries sont presque complètes, avec des pièces présentes même en plusieurs exemplaires, alors que d'autres sont vides. L'analyse des documents issus de ce fonds laisse donc certaines questions sans réponse. Surtout, étant donné l'importance des transformations survenues sur le site au cours des années, il est parfois difficile de distinguer entre un projet élaboré mais non réalisé et un projet réellement mis en œuvre puis modifié ou démoli ultérieurement.

3- Voir Centre des archives historiques de la SNCF [désormais : SNCF, CAH], Archives de la Compagnie du chemin de fer de Paris à Orléans, 1838-1938. Répertoire méthodique dressé par Guillaume Roussean sous la direction de Christian Perrot, juin 2000.

4- Ce fut le cas de presque toutes les archives des services « Voie et Bâtiments » ou « Voie et Travaux » des anciennes compagnies de chemin de fer; les archives de ces services furent souvent conservées dans les gares. La SNCF réunit depuis 1995 ce type de documents dans son Centre des archives historiques au Mans. 
Il est important aussi de noter que l'image qui ressort de notre documentation exprime avant tout le point de vue de l'exploitant ferroviaire sur l'ouvrage que nous avons étudié. Ce qui intéresse la Compagnie du Paris-Orléans n'est pas la rue Watt en elle-même mais les ponts ferroviaires qui l'enjambent. Le vocabulaire utilisé pour désigner ces ponts est déterminé par référence à la voie ferrée : l'expression si souvent rencontrée, "P.I. de la rue Watt», renvoie à un passage ou "pont inférieur », situé au-dessous des voies ferrées qu'il porte. Nous verrons qu'il était un certain temps question de transformer l'ouvrage en «P.S. » ou "pont supérieur », c'est-à-dire en un pont passant audessus des voies ferrées et portant la chaussée publique sur son tablier. La désignation de sa localisation, « P.K. 2,008 », renvoie au " point kilométrique », la distance en kilomètres depuis la tête des voies.

\section{Le contrôle du ministère des Travaux publics}

Le site étudié met en contact la Compagnie du Paris-Orléans et la ville de Paris. Mais elles n'étaient pas seules impliquées dans les projets d'aménagement qu'il fallait définir et mettre en œuvre. La Compagnie d'Orléans était assujettie au contrôle du ministère des Travaux publics et son activité d'aménagement n'échappait pas à cette règle.

Le contrôle de l'exploitation des chemins de fer constituait en effet une activité considérable au sein du ministère des Travaux publics, souvent modifiée et réorganisée avec l'évolution de la législation et de la réglementation régissant les relations entre les compagnies et l'État.

«Le développement progressif des voies ferrées et le rang généralement élevé des ingénieurs de l'État placés par les Compagnies à la tête de leur exploitation ont déterminé le Gouvernement à mettre au sommet de la hiérarchie du contrôle, des fonctionnaires jouissant d'une grande autorité par leur âge, leurs talents et leur grade dans le Corps des Ponts et Chaussées ${ }^{5}$. »

À la fin du XIX ${ }^{e}$ siècle, il existait ainsi au ministère une direction du Contrôle pour chaque réseau, avec à sa tête un inspecteur général des ponts et chaussées ayant sous ses ordres une hiérarchie d'ingénieurs en chef, d'ingénieurs ordinaires et de conducteurs de travaux.

"Chaque directeur a auprès de lui : $1^{\circ}$ un ingénieur en chef des ponts et chaussées, chargé du contrôle des travaux et du mandatement général des dépenses du contrôle ; $2^{\circ}$ un ingénieur en chef des ponts et chaussées, chargé du contrôle de l'exploitation technique $; 3^{\circ}$ un ou deux inspecteurs principaux de l'exploitation commerciale ${ }^{6} . »$

5- Alfred Picard, Traité des chemins de fer, Paris, J. Rothschild éditeur, 1887, t. III, p. 152. 6- Ibidem. 


\section{Le temps long des projets ferroviaires}

Nous verrons que l'aménagement de la rue Watt soulevait des problèmes d'alignement qui ont fait l'objet d'un conflit entre la Compagnie du Paris-Orléans et la ville de Paris qui a duré presque trente ans.

Les documents analysés montrent que la Compagnie du ParisOrléans élaborait en effet des projets sur de longues durées, trente ou quarante ans. C'est ainsi que nous voyons la compagnie présenter un projet à l'approbation du ministère au début des années 1870 concernant l'agrandissement de la gare des marchandises à Ivry - un projet qui impliquait la suppression du pont sur la rue Watt du raccordement intra-muros du chemin de fer de Ceinture. On peut considérer que ce projet, après de nombreuses modifications et améliorations, est pour sa majeure partie réalisé en 1913. Il s'agit d'une période qui a vu des évolutions radicales auxquelles la gare a dû s'adapter, plus particulièrement pendant les premières années du XXe siècle : la prolongation de ses lignes jusqu'à la nouvelle gare quai d'Orsay, les débuts de l'électrification du réseau (d'abord entre Austerlitz et Orsay en 1900, puis entre Paris et Juvisy en 1904) ainsi que le doublement des voies entre Paris et Brétigny.

Mais malgré ces transformations profondes dans les conditions d'exploitation de la gare, nous verrons la compagnie présenter en 1912 un « projet d'avenir lointain » qui est sous certains aspects une prolongation du projet des années 1870 : il s'agissait de réorganiser le plateau de voies, en déplaçant notamment les ateliers de réparation et les services des messageries. Ce projet, conçu pour être mis en œuvre selon un phasage comportant des étapes qui ne seraient réalisées que « si nécessaire », est achevé dans sa totalité en 1929 quand il fait l'objet d'une importante publication dans la Revue générale des chemins de fer (fig. 4).

\section{Historique : les principales étapes}

Certains faits fondamentaux concernant l'histoire des ponts de la rue Watt sont rapportés dans des documents qui accompagnent les épreuves rendues obligatoires par une circulaire ministérielle de 1891, lors desquelles on devait noter, en plus des résultats des épreuves et vérifications techniques entreprises, des informations concernant l'histoire de l'ouvrage, l'origine de ses matériaux, ainsi que les entreprises responsables de sa construction ${ }^{7}$. Cependant, ces quelques faits fondamentaux ne permettent pas à eux seuls de comprendre une histoire qui, au vu des autres documents examinés, parait en fait assez complexe.

7- SNCF, CAH 236LM30/4/5;236LM27/6/2;236LM30/4/6/8;236LM27/6/3. Chemin de fer d'Orléans. Service de la Voie. Pont inférieur de la rue Watt [...] Dossier prescrit par C.M. du 19 août 1891. 

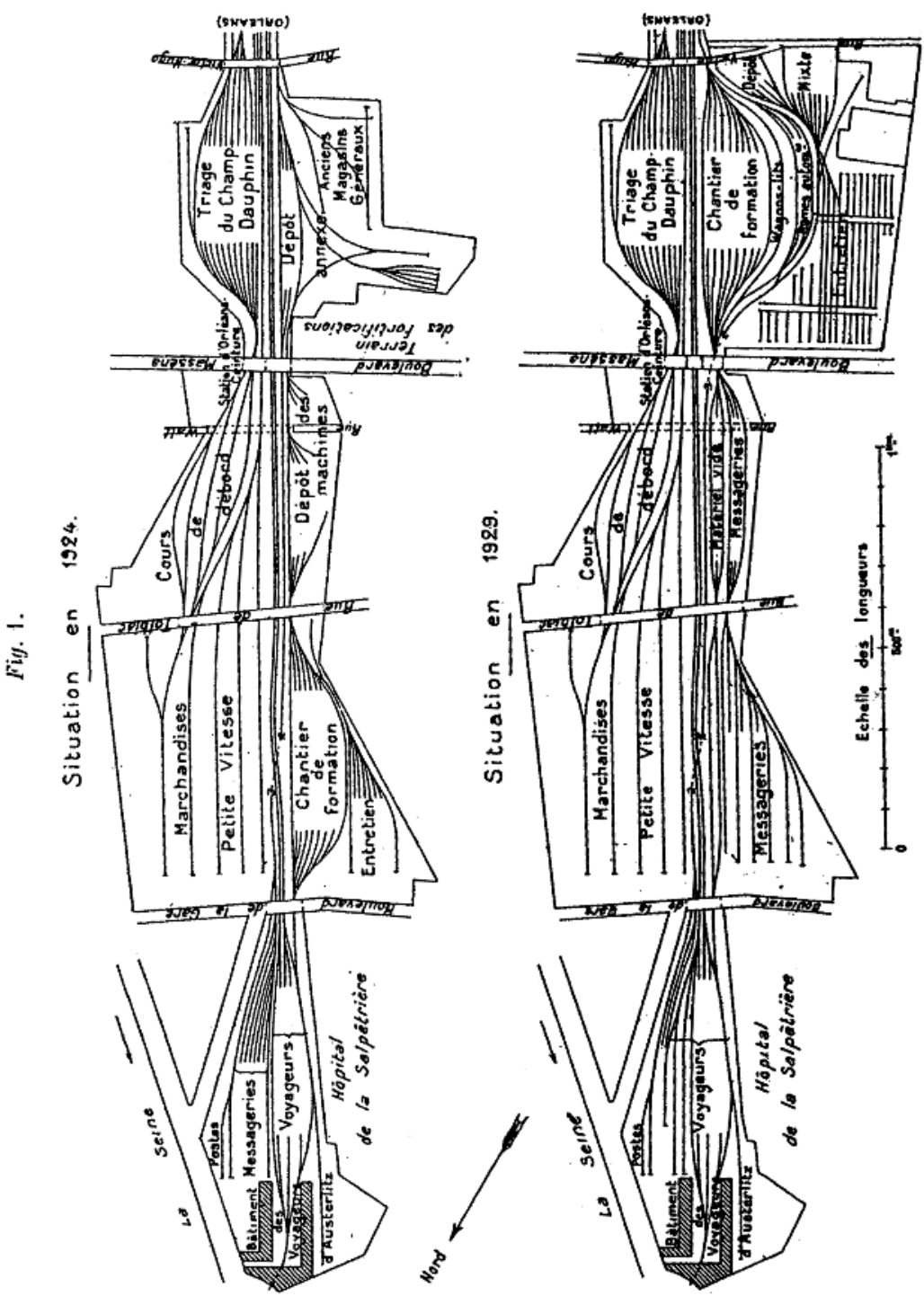

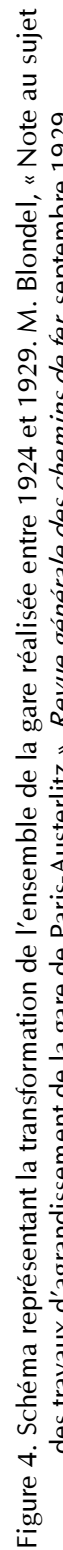




\section{Les origines}

On apprend ainsi que les origines du pont remontent aux débuts mêmes du chemin de fer sur le site, en 1839 : les rails auraient alors enjambé la rue portés par des poutres en bois, le pont ayant une portée de 3 mètres sur une longueur de 8 mètres. En 1854, ce pont aurait été remplacé par un ouvrage plus important, avec un tablier métallique et une portée de 5 mètres sur une longueur de 24 mètres, ouvrage allongé à 43 mètres en 1867 mais en conservant la même portée de 5 mètres. Cette période de l'histoire de l'ouvrage est évoquée dans des documents ultérieurs, où l'on souligne que pendant les années 1850 la rue Watt, située dans une partie de la commune d'Ivry qui n'avait pas encore été annexée à Paris, «n'était que le chemin de la Croix-Jarry et ne débouchait pas sur le quai de la gare $»^{8}$. C'est également pendant les années 1850 que fut réalisé un autre pont sur la rue Watt, portant le raccordement entre le chemin de fer d'Orléans et la Ceinture' : ce pont était situé vers l'extrémité Seine de la rue Watt, à quelques mètres du premier pont $\mathrm{PO}$ (fig. 5).

\section{0 années pour régulariser un alignement}

C'est au cours des années 1870 que des projets plus ambitieux pour ces ouvrages sont élaborés, dans le cadre d'un important programme de remaniement des voies PO. Il s'agissait d'agrandir la gare des marchandises d'Ivry et de déplacer extra-muros le raccordement avec la Ceinture : les terrains occupés par le raccordement intra-muros devaient ainsi être libérés pour l'extension de la gare. Notons que, selon ce projet, le nouveau raccordement avec la Ceinture, maintenant situé extra-muros, devait passer sur le boulevard Masséna par un passage à niveau, solution qui, comme nous pouvons l'imaginer, allait faire l'objet d'importantes réserves par la suite ${ }^{10}$. Des décisions ministérielles de 1873

8- SNCF, CAH 40LM34/1/4: Conseil Municipal de Paris. 1911. Rapport de la $3^{\mathrm{e}}$ Commission, relatif au raccordement extra-muros du réseau d'Orléans avec le chemin de fer de Ceinture, avec traversée souterraine du boulevard Masséna, présenté par M. J. Hénaffe Conseiller Municipal.

9- Le chemin de fer de Grande Ceinture fut concédé en 1875 et sa mise en service par tronçons s'étale entre 1877 et 1886 . Avant cette date, la «Ceinture » est donc la future Petite Ceinture, mais elle ne fait pas encore le tour complet de Paris : on distingue entre Ceinture Rive droite et Ceinture Rive gauche. Le PO participait au syndicat concessionnaire du chemin de fer de Ceinture Rive droite, ouvert à l'exploitation par sections entre 1852 et 1859. Voir Alfred Picard, Les Chemins de fer français. Étude historique sur la constitution et le régime du réseau, t. I à VI, Paris, J. Rothschild, 1885.

10- SNCF, CAH 40LM29/32/6/3 : Agrandissement des gares aux marchandises d'Ivry. Modification des ponts de la rue Watt. Procès-verbal de conférences. 16 septembre 1897 ; 6 mai 1898 ; 21 juin 1898 ; 18 juillet 1898 . 


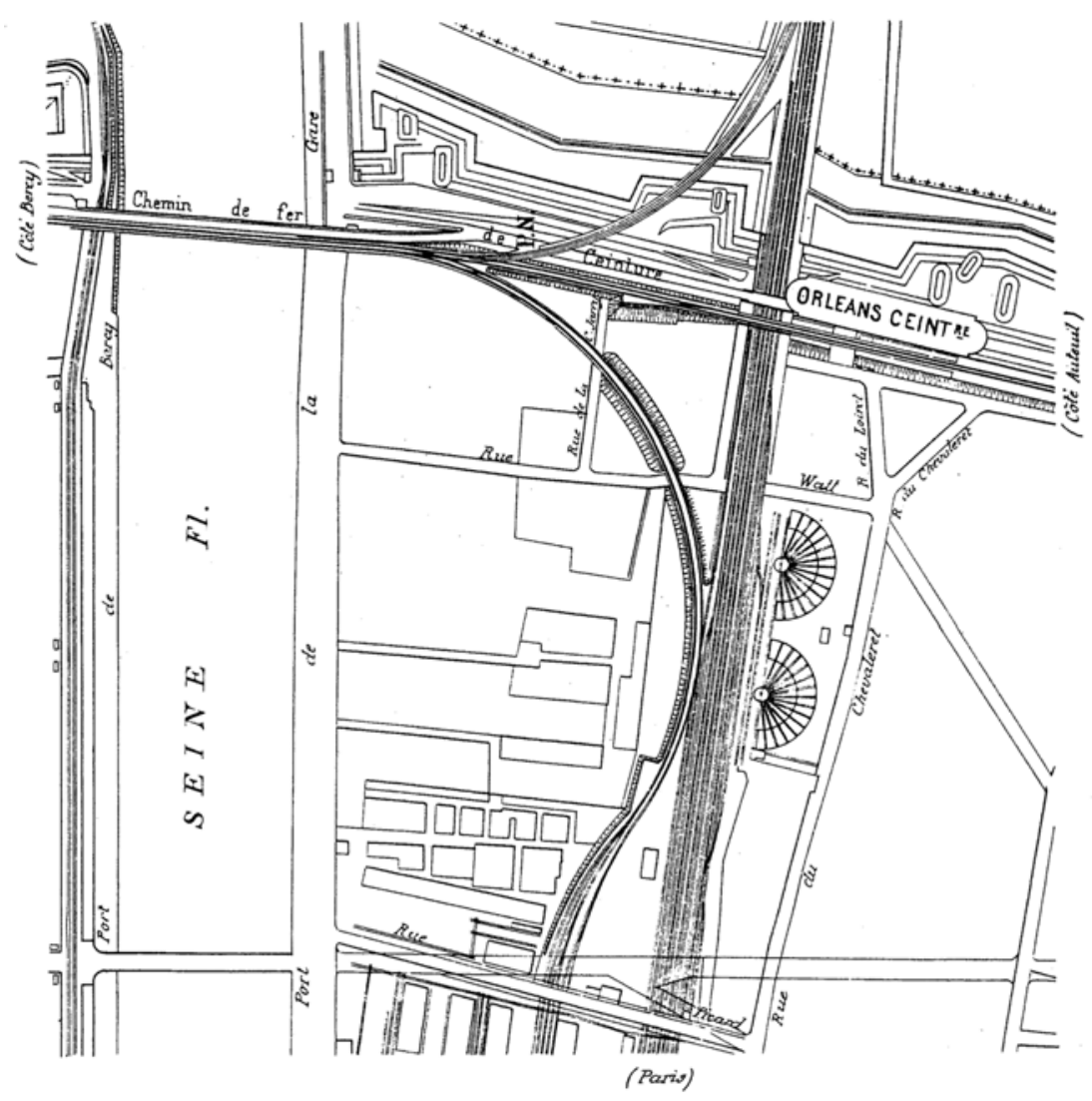

Légende

Bleu $\square$ Domaine du Chemin de fer de Ceinture (R.D.).

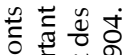

능 의

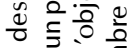

○드.

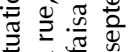

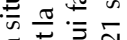

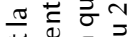

ㄴํㄴ

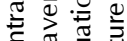

产

즈

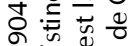

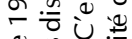

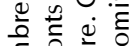

है

눙 $\frac{1}{2}$

ธ०

恼这

过

过

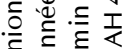

的记

는

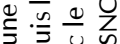

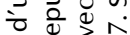

중. 종

迹. 焉

iे

造

흘 岂

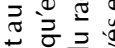

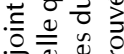

这这

焉造

कू ญ.

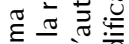

泮

议

เก

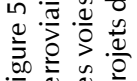

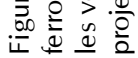




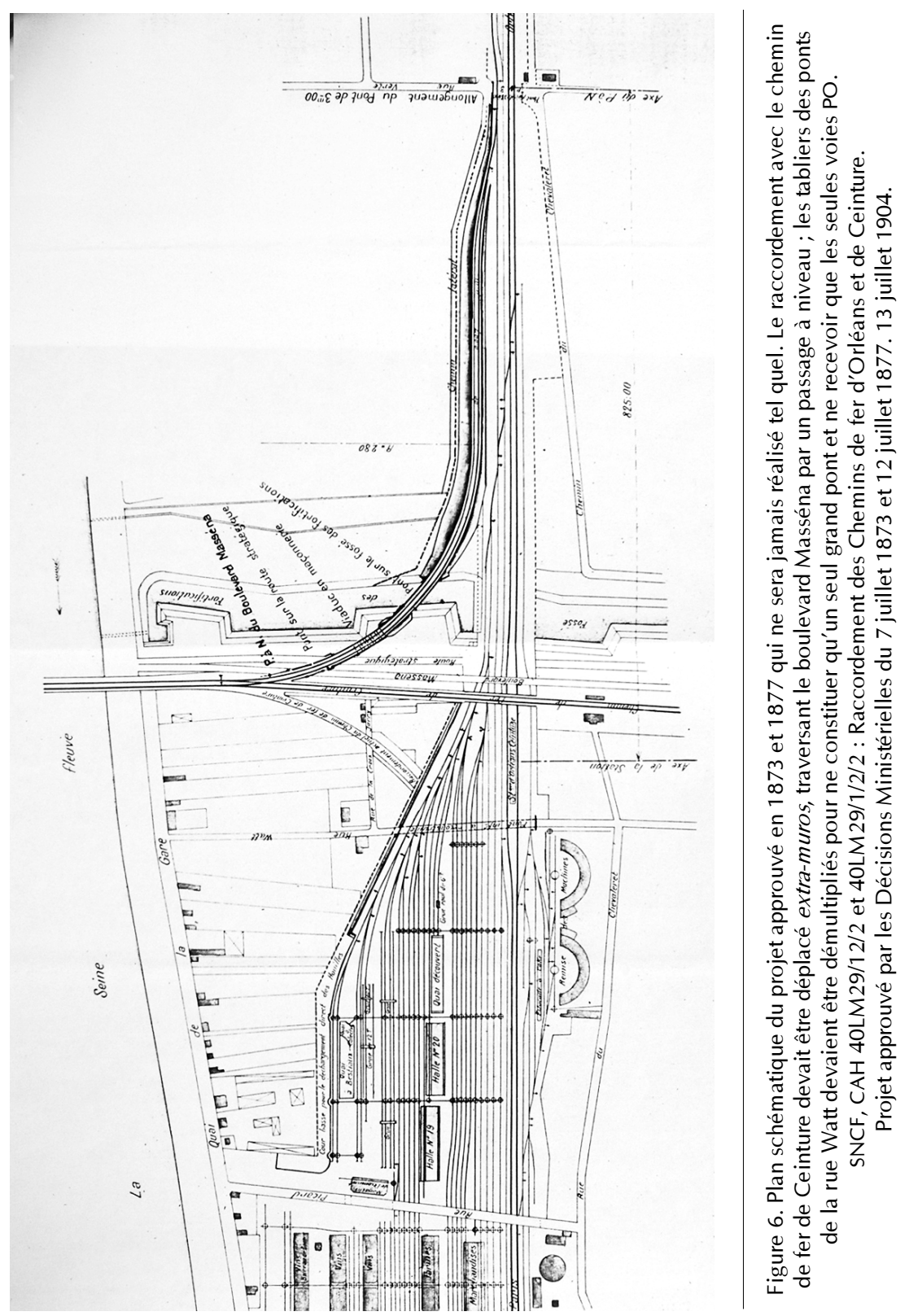


et 1877 ont cependant autorisé le PO à mettre en œuvre ce projet, les ponts sur la rue Watt devant être remplacés par un ouvrage d'une ouverture de 12 mètres (et non plus 5) sur une longueur de 125 mètres (fig. 5 et fig. 6).

La mise en œuvre de ce projet a été commencée mais jamais achevée. La Compagnie d'Orléans s'est approvisionnée en poutres et pièces de pont en fer afin de construire un tablier de 12 mètres de portée sur 125 mètres de longueur, mais cet ouvrage ne fut réalisé que sur 71 mètres, entre 1881 et 1883. La construction du nouveau pont a semble-t-il procédé à partir de la rue du Chevaleret, pour s'arrêter à quelques mètres du pont du raccordement intra-muros avec la Ceinture, qui traversait par ailleurs cette rue à un niveau légèrement plus élevé que ne le faisaient les voies PO.

Les raisons de cette exécution incomplète d'un ouvrage approuvé et dont les matériaux avaient été approvisionnés ne sont explicitées que partiellement par les documents étudiés. En 1904, pour expliquer cet arrêt des travaux, on évoquera une baisse de trafic à la gare d'Ivry ainsi que des «difficultés de service que l'on craignait par suite de la suppression du raccordement intérieur de la Ceinture $»^{11}$. Lors des discussions organisées à la fin des années 1890 entre la Compagnie du Paris-Orléans, la ville de Paris et le service de contrôle du réseau PO au ministère des Travaux publics, le directeur de la compagnie avait proposé des explications à peine plus détaillées. Il affirme dans un exposé du 6 mai 1898 que ce n'était que pendant l'exécution du projet de 1874 que la compagnie s'était rendu compte des difficultés qui pourraient être provoquées par la suppression du raccordement intra-muros avec la Ceinture, tant pour les trains de marchandises que pour ceux des voyageurs, et que la décision avait été prise alors d'ajourner la démolition du pont existant.

On peut cependant supposer que la Compagnie d'Orléans n'avait aucun intérêt à construire un nouveau pont à l'emplacement du raccordement intra-muros avec la Ceinture tant que ce raccordement n'avait pas été effectivement déplacé et qu'elle n'était pas en mesure d'arrêter la manière dont ses voies allaient désormais traverser la rue Watt. Le mode de mise en œuvre du nouveau pont dépendait en effet de l'angle selon lequel les voies devaient traverser la rue, déterminant la disposition des poutres portant le tablier du pont et les rails.

11- SNCF, CAH 40LM29/1/2/1: Agrandissement de la gare aux marchandises et raccordement extra-muros avec le chemin de fer de Ceinture. Notice explicative. 13 juillet 1904. 
Quelles qu'en aient été les raisons, le pont de 1881-1883 ne fut donc réalisé qu'à moitié, laissant le raccordement avec la Ceinture sur le pont existant de 5 mètres de portée, et la compagnie en possession de poutres en fer en réserve. La rue Watt elle-même était laissée avec un alignement irrégulier, avec un point d'étranglement sous le pont du raccordement, où elle passait donc de 12 mètres à 5 mètres de largeur (fig. 7, fig. 8) Cette situation, qui n'a pas manqué de provoquer des réactions de la part des ingénieurs de la ville de Paris, a duré 30 ans, jusqu'en 1913, quand la Compagnie d'Orléans a enfin ajouté trois tabliers neufs à son pont existant et régularisé l'alignement de la rue Watt.

Cette partie de l'ouvrage a néanmoins fait l'objet de quelques améliorations pendant les années 1880 et 1890 . On sait par exemple qu'en 1884, "pour donner satisfaction à une demande de M. le Préfet de la Seine ", la compagnie a mis en place un platelage en tôle striée dans les entrevoies adjacentes à certaines voies; et que, en 1887, on a établi des planchers en bois " pour empêcher l'huile et l'eau provenant des machines de tomber sur les passants $»^{12}$. Il s'agit là d'interventions résultant donc de pressions exercées par la ville de Paris pour obtenir que la compagnie assume la responsabilité des effets sur la voie publique de son exploitation des ponts ferroviaires.

\section{Entre compagnie ferroviaire et municipalité}

En 1896, les étais en bois soutenant le bardage du remblai d'une des culées du pont du raccordement avaient fait l'objet d'une plainte du commissaire voyer du XIII ${ }^{\mathrm{e}}$ arrondissement (fig. 7, fig. 8). En effet, lors du même exposé du 6 mai 1898 évoqué plus haut ${ }^{13}$, le directeur de la compagnie explique à l'ingénieur chargé du contrôle du réseau PO au ministère des Travaux publics que, même si «les étais en bois qui soutiennent les terres » du remblai de la culée du pont du raccordement étaient « en bon état», la compagnie était disposée «à remplacer ces étais et le bardage en bois par un mur en maçonnerie ». Il affirme que les plaintes concernant l'irrégularité de l'alignement de la rue Watt «ne se sont manifestées qu'en 1896 et 1897 », et qu'elles n'ont "aucun caractère d'acuité ». Selon la compagnie, «la gêne apportée aux voitures par l'étranglement de la rue Watt » pouvait être « provisoirement acceptée, étant donnée la faible importance de la circulation qui emprunte cette rue ». Le directeur explique par ailleurs que « pour donner satisfaction aux plaintes », la compagnie avait élaboré des projets « tendant à [...]

12- Voir les documents cités note 7.

13- Voir note 10. 

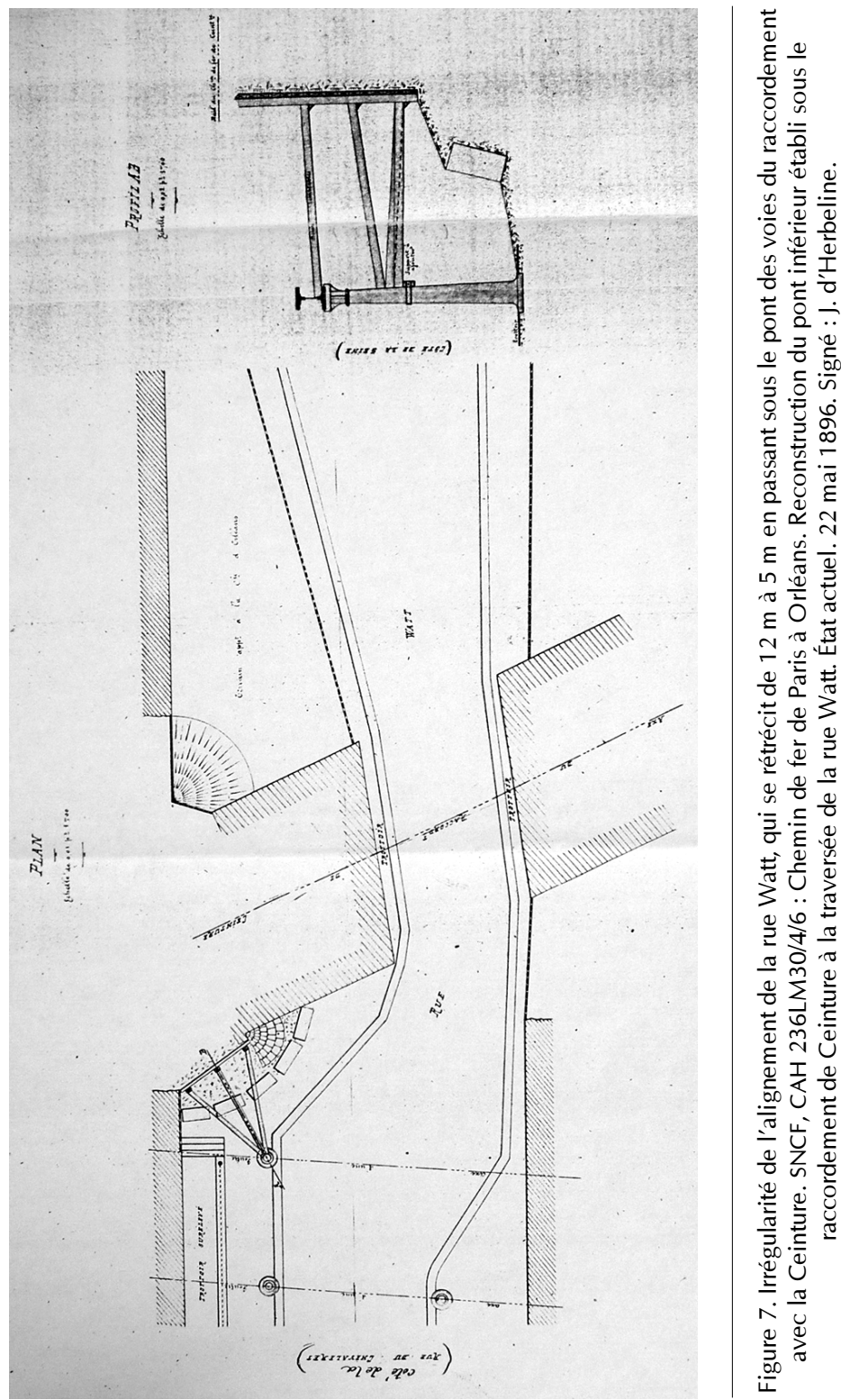


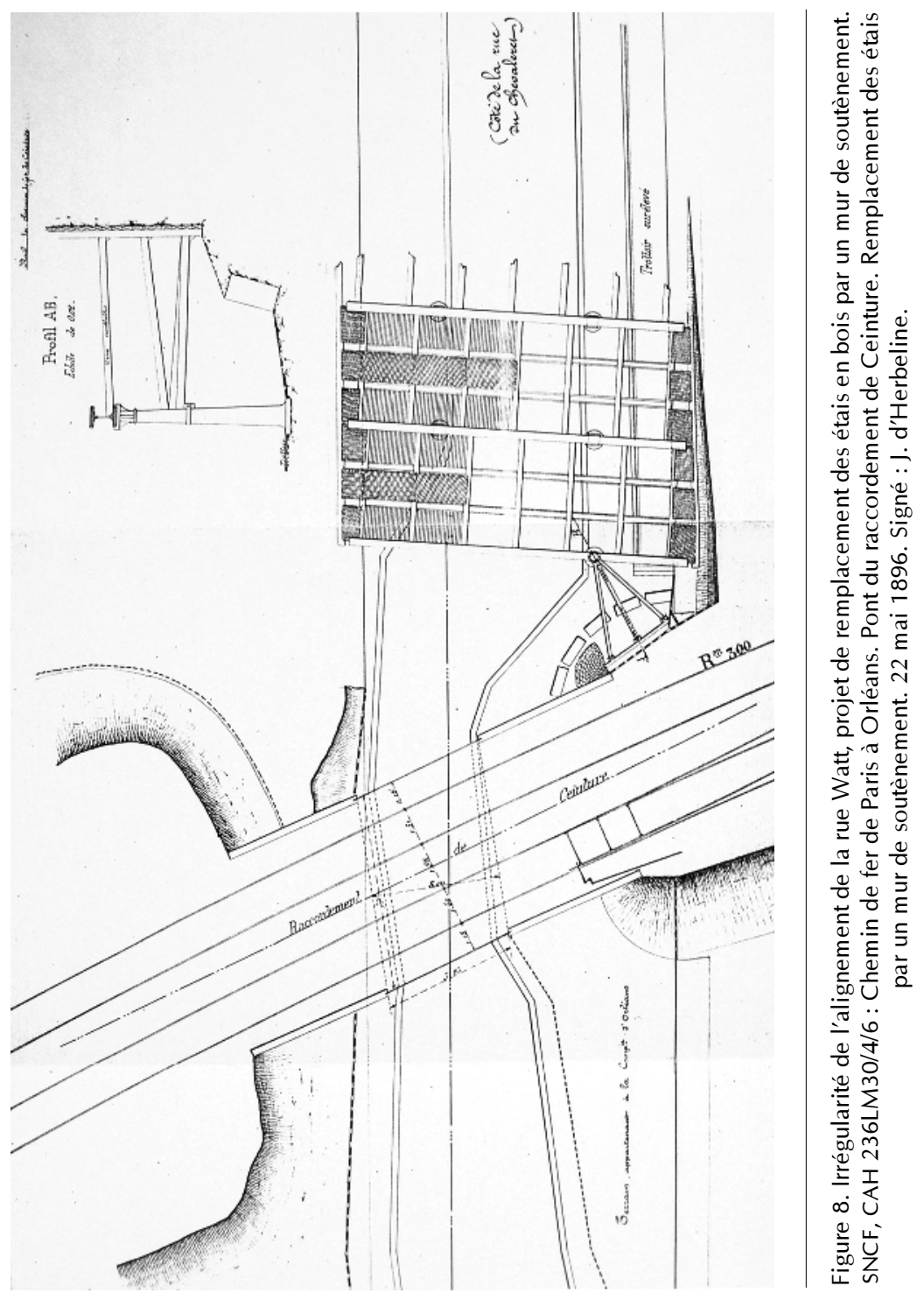


substituer au passage actuel un pont nouveau de 12 mètres de portée » : il s'agit sans doute des projets signés par l'ingénieur Jégou d'Herbeline le 22 mai 1896, qui accompagnaient les projets de la même date visant à remplacer les étais en bois par un mur de soutènement (fig. 7, 8). Le directeur affirme que la compagnie aurait demandé d'elle-même l'autorisation de réaliser le nouveau pont « si le prolongement prochain de notre ligne jusqu'au quai d'Orsay n'était pas venu transformer le problème »; il ajoute que « le doublement plus ou moins prochain de nos voies aux abords de Paris » aurait lui aussi rendu nécessaire une modification importante du tracé du raccordement avec la Ceinture.

La gravité du problème posé par le goulet d'étranglement sous le pont de raccordement fait manifestement l'objet de perceptions différentes de la part des ingénieurs de la ville de Paris et de celle de leurs collègues de la Compagnie d'Orléans. Lors des exposés sur ces questions organisés en 1897 et $1898^{14}$, les ingénieurs de la ville de Paris insistaient sur le fait que le décrochement dans le tracé de la rue Watt constituait un réel danger pour la circulation. « Les voitures qui viennent du côté de la Seine ne peuvent apercevoir celles qui viennent de l'autre côté, et n'en sont pas aperçues davantage ». L'ingénieur en chef de la voie publique souligne encore en 1898 le caractère « vraiment inacceptable du point de vue de la voie publique » de la saillie dans l'alignement de la rue.

\section{Arbitrages du service du contrôle au ministère des Travaux publics}

Lors de ces conférences organisées en 1897 et 1898, le ministère des Travaux publics devait trancher entre les points de vue divergents de la Compagnie d'Orléans et de la ville de Paris. L'ingénieur ordinaire Rousseau donne un premier avis en 1898 ; le dernier mot revient à M. d'Ussel, ingénieur en chef du contrôle du réseau d'Orléans. Pour M. Rousseau, même si la rue Watt est peu fréquentée, l'étranglement formé par les culées du pont du raccordement «n'est pas sans danger». Il comprend que la compagnie souhaite ajourner la réalisation d'un nouveau pont sur ce point, qui « risquerait de n'être utile que pendant une durée très limitée à cause des modifications que peut subir le raccordement ». Il lui semble néanmoins que « cette amélioration réclamée par la ville et approuvée depuis 1874 a été suffisamment ajournée ». Sa conclusion est « d'inviter la Compagnie d'Orléans à présenter un projet qui fasse disparaitre le défaut de largeur du pont de la rue Watt». L'avis de son supérieur hiérarchique au ministère des Travaux publics est cependant plus nuancé et, surtout, moins contraignant pour la compagnie : il conclut

14- Ibid. 
en invitant cette dernière non pas à « présenter un projet » mais à « étudier un projet », qui " procurerait à la rue Watt l'amélioration réclamée ».

\section{Des collaborations difficiles}

Curieusement, les conclusions de ces conférences de 1897 et 1898 semblent être restées comme en suspens pendant trois années. Le préfet de la Seine les adresse au ministre des Travaux publics en septembre 1901 seulement, le retard étant dû à « un malentendu entre le Service Municipal et le Service de Contrôle ».

Dans sa lettre d'envoi du dossier de septembre 1901, le préfet prie le ministre de «vouloir bien inviter la Compagnie d'Orléans à procéder dans le plus bref délai à l'étude du projet de reconstruction du viaduc de la rue Watt ${ }^{15}$. Cette lettre relançant l'affaire semble avoir eu un certain effet, puisqu'on trouve un nouveau projet, élaboré en 1902, de «Reconstruction du pont sur la rue Watt à la traversée du raccordement avec la Petite Ceinture ». Cependant, en janvier 1903, la compagnie écrit à son tour au ministre des Travaux publics en proposant de ne pas donner suite aux projets ayant fait l'objet des conférences de 1897-1898; elle affirme que de nouveaux projets sont en cours d'élaboration « entraînant très-probablement pour la rue Watt la réalisation du programme de $1874 »^{16}$, affirmation répétée plus tard la même année dans une lettre du directeur de la compagnie à l'inspecteur du service municipal de la ville de Paris.

Mais le ministre semble maintenant s'impatienter. En février, juin et août 1903, il réclame avec de plus en plus d'insistance la communication du projet d'amélioration de la rue Watt. Sa lettre du 8 août adressée à «MM. les administrateurs de la Compagnie d'Orléans » est la plus sèche :

«Par plusieurs lettres restées sans réponse, j’ai invitée votre Compagnie à m'adresser d'urgence le projet d'amélioration des installations de la gare d'Ivry, y compris la reconstruction définitive et la mise à l'alignement des ponts de la rue Watt. Je ne m'explique pas, Messieurs, le silence de votre de Compagnie, qui aurait pu, si elle n'était pas en mesure de fournir le projet réclamé, faire connaître à mon Administration la cause de cet empêchement. Je vous prie de me donner, à cet égard, des explications dans le plus bref délai possible ${ }^{17}$.»

15- SNCF, CAH 236LM30/4/4/3 : Lettre du Préfet de la Seine au Ministre des Travaux publics. 24 septembre 1901.

16- SNCF, CAH 40LM29/32/6/1: Brouillon de lettre. Directeur de la Compagnie à M. David, ingénieur de Contrôle du réseau Paris-Orléans au Ministère des Travaux publics. 13 janvier 1903.

17- SNCF, CAH 40LM29/32/2: Lettre du Ministre des Travaux publics à MM les Administrateurs de la Compagnie d'Orléans, Paris le 30 juin 1903. 
Le directeur de la compagnie lui répond le 13 août que «nous venons de terminer l'étude de notre projet [...] comportant la reconstruction définitive et la mise en alignement du pont de la rue Watt $»^{18}$.

Cependant, il écrit aussi que ce nouveau projet ne sera soumis à l'approbation du ministre qu'une fois pris l'avis du Syndicat de la Ceinture, puisque le nouveau projet comporte la substitution d'un raccordement extra-muros à celui existant intra-muros. Nous n'avons pas vu d'écrit qui explique pourquoi cet aspect du projet, qui existait déjà dans celui qui avait été approuvé en 1874, devait recevoir à nouveau l'aval du Syndicat de la Ceinture ; aval qui lui sera en effet refusé, non pas par le Syndicat de Ceinture lui-même mais par l'ingénieur du contrôle du syndicat au ministère. Le directeur de la compagnie affirme néanmoins au ministre que « nous pouvons prendre d'ores et déjà l'engagement de réaliser la mise en alignement de la rue Watt dans les conditions désirées par la ville de Paris $»^{19}$.

À l'intérieur de la compagnie, cependant, le processus d'étude et d'approbation du projet par chacun des services prend du temps. Le service de la voie et des travaux réclame encore fin novembre 1903 les observations du service de l'exploitation sur cette étude de la « Régularisation des alignements de la rue Watt par la suppression du pont établi pour le passage du raccordement avec la Ceinture ». Un plan d'ensemble du pont de la rue Watt daté du 12 décembre 1903 représente le pont du raccordement exactement tel qu'il était depuis les années 1850 (fig. 9).

\section{Un problème qui se complique}

Le problème posé par le pont du raccordement sur la rue Watt était effectivement lié à celui du remplacement du raccordement intramuros par un nouveau raccordement extra-muros. Comme nous l'avons mentionné, dans le projet initial de 1874 le nouveau raccordement devait traverser le boulevard Masséna par un passage à niveau, solution que paraissait de moins en moins souhaitable avec le passage du temps. En 1904, la compagnie modifie ses études sur cette partie du projet en substituant des ponts à ce passage à niveau. L'ingénieur responsable de l'élaboration de ces études, M. Jégou d'Herbeline, écrit en juin 1904 à son supérieur hiérarchique en suggérant de séparer la question des ponts de la rue Watt du reste du projet :

«Il est à craindre que les décisions à prendre sur toutes ces questions de modifications de la gare d'Ivry et du raccordement ne durent bien longtemps. Suivant moi il conviendrait de régler de

18- SNCF, CAH 40LM29/32: Brouillon de lettre. Directeur de la Compagnie à M. le Ministre des Travaux publics. 13 août 1903.

19- Ibid. 


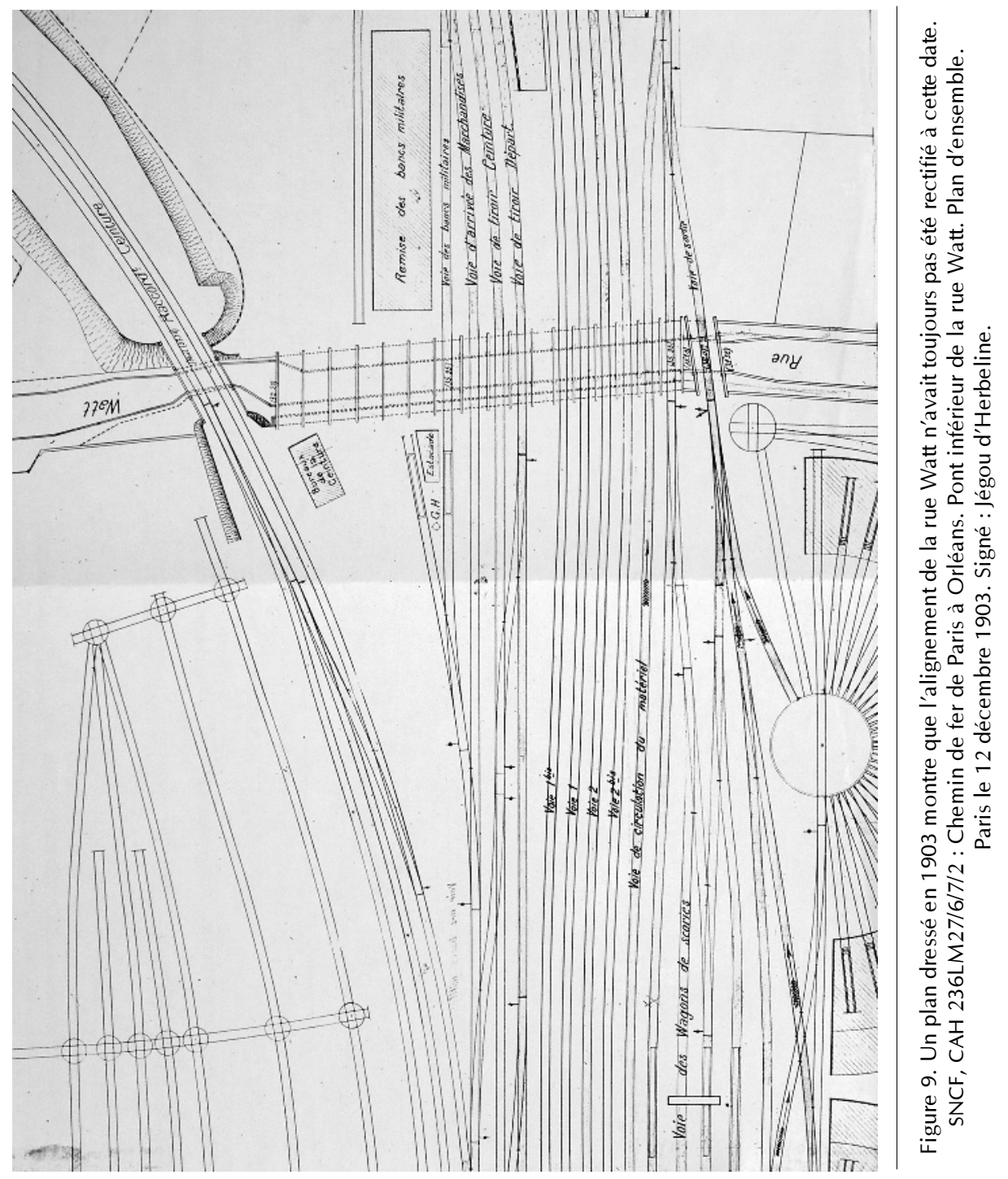


suite la question de la rectification de la rue Watt, en faisant un pont provisoire sous le raccordement actuel de ceinture; il y aurait peu de dépenses perdues puisque les culées seraient utilisées plus tard ainsi que le tablier ${ }^{20}$. »

Notons que, comme nous l'avons vu, M. Jégou d’Herbeline avait déjà élaboré deux projets non réalisés pour le remplacement du pont de raccordement de la rue Watt, l'un en 1896 et l'autre en 1902.

Dans le courant de l'année 1904, le ministère approuve l'allongement, souhaité par la compagnie, des ponts de la rue Watt du côté de la rue du Chevaleret, dans le cadre du dédoublement des voies entre Paris et Brétigny. Un nouveau tablier, réalisé avec les poutres métalliques approvisionnées en 1881 mais jamais utilisées, est construit pour la nouvelle voie de sortie du dépôt en 1905 : il s'agit vraisemblablement du projet daté du 12 décembre 1903 mentionné plus haut ${ }^{21}$. Mais même si le ministre avait approuvé en avril ce projet de nouveau tablier côté rue du Chevaleret, en mai il demande à nouveau « dans le plus bref délai possible » le projet comportant la reconstruction définitive et la mise en alignement de la rue Watt.

La compagnie ne semble cependant pas disposée à suivre la suggestion, émise par M. Jégou d’Herbeline en juin 1904, de séparer le problème de la rue Watt du reste du projet concernant le raccordement extra-muros et le remplacement par un pont du passage à niveau initialement prévu sur le boulevard Masséna. Au contraire, la solution à donner au problème du tracé de la rue Watt est clairement intégrée au projet d'ensemble pour l'agrandissement de la gare aux marchandises et le raccordement extra-muros avec la Ceinture, présenté dans une notice explicative en juillet 1904. «Le moment est donc venu d'attaquer une nouvelle phase en vue de la réalisation du programme de 1873 et d'opérer le déplacement du raccordement de la Ceinture et l'achèvement de la rue Watt. ${ }^{22}{ }^{\prime}$

Ce projet fera l'objet de conférences du même type que celles organisées en 1897-1898 pour recueillir les avis des divers services et administrations intéressés par les aménagements proposés : ici, il s'agit de l'ingénieur du service municipal de la ville de Paris, de l'ingénieur du contrôle de la Ceinture au ministère et du service du Génie du gouvernement militaire de Paris (le projet touchant aux fortifications de la ville).

20- SNCF, CAH 40LM29/22: Notes manuscrites, 28 mars et 9 juin 1904.

21- Ibid.

22- SNCF, CAH 40LM29/1/2/11: Agrandissement de la gare aux marchandises et raccordement extra-muros avec le chemin de fer de Ceinture. Notice explicative. 3 juillet 1904. 
Ouvertes en août 1904 elles dureront jusqu'en juillet 1906, étant donné la complexité du projet de traversée du boulevard Masséna et du raccordement des voies de la Ceinture avec celles, modifiées depuis 1874, du PO. Mais en ce qui concerne la rue Watt, les seules réserves de l'ingénieur du service municipal concernent des points de détail ; le chef du Génie n'en exprime aucune. C'est l'ingénieur du contrôle de la Ceinture au ministère qui, comme nous l'avons mentionné, s'oppose au projet, insistant sur le fait que « le raccordement nouveau ne remplacera pas du tout l'ancien du point de vue des voyageurs $»^{23}$.

\section{Des améliorations pour I'exploitation ferroviaire, enfin une solution pour la ville}

La compagnie finit par obtenir l'approbation de l'ingénieur du Contrôle de la Ceinture au raccordement extra-muros, en répondant point par point à ses objections. Mais le pont sur la rue Watt restera néanmoins en place jusqu'en 1913. En attendant, la compagnie procède à l'allongement de ses ponts dans l'autre partie de la rue, ajoutant en 1908 encore un autre tablier côté rue du Chevaleret pour relier une nouvelle remise avec pont tournant de $23 \mathrm{~m} 50$ avec une des deux rotondes existantes : là encore, le tablier sera réalisé avec les poutres en fer qui avaient été approvisionnées en 1881.

En avril 1911, le projet présenté par la compagnie pour assurer la modification de sa gare et le raccordement extra-muros avec la Ceinture est examiné par la commission voirie du conseil municipal de Paris ${ }^{24}$. La partie du projet concernant les ponts de la rue Watt ne soulève aucune objection, au contraire. Fin novembre 1912, l'entrepreneur Boisfard, à Issoudun, signe le cahier des charges spécial portant sur l'établissement de trois tabliers métalliques sur la rue Watt et l'alignement de la rue Watt sera enfin normalisé en $1913^{25}$.

23- SNCF, CAH 40LM29/4/2, 4, et 5 : Préfecture du département de la Seine. Chemin de fer d'Orléans. Ligne de Paris à Orléans. Gare d'Ivry. Agrandissement du Service des Marchandises. Raccordements des Chemins de fer d'Orléans et de Ceinture. Procèsverbal de conférence mixte. 7 juillet 1906.

24- SNCF, CAH 40LM34/1/4: Conseil Municipal de Paris. 1911. Rapport de la $3^{e}$ Commission, relatif au raccordement extra-muros du réseau d'Orléans avec le chemin de fer de Ceinture, avec traversée souterraine du boulevard Masséna, présenté par M. J. Hénaffe Conseiller Municipal.

25- SNCF, CAH 236LM27/6/4 : Chemin de fer de Paris à Orléans. Service de la Voie et des Travaux. Raccordements extra-muros des chemins de fer d'Orléans et de Ceinture. Projet de modifications. Cahier des charges spécial. 18 novembre 1912. 


\section{Le projet de supprimer la rue Watt}

Curieusement, ce n'est que peu de temps après cette régularisation si longtemps attendue par la ville que l'idée émerge parmi les ingénieurs de la compagnie de faire supprimer purement et simplement la rue Watt. C'est M. Balling, ingénieur en chef du $1^{\text {er }}$ arrondissement ${ }^{26}$, qui semble être à l'origine de cette proposition. Il explique dans une lettre du 4 juillet 1917 à l'ingénieur en chef de la Voie et des Travaux, M. Couvrat-Desvergnes, que selon le «plan d'avenir lointain » dressé en 1912 par la commission d'extension des gares de Paris et prévoyant le déplacement extra-muros du dépôt, environ 15000 mètres de terrains serait laissés inutilisés « à cause de la nécessité de ménager la rampe par laquelle la rue Watt descend sous nos voies ». Par ailleurs, avec l'inflation des coûts des matériaux de l'époque, la construction de nouveaux tabliers entraînerait une « dépense considérable »; ces sommes pourraient être économisées et les 15000 mètres de terrains exploités si la rue Watt était supprimée ${ }^{27}$.

Dans une représentation schématique des agrandissements réalisés pendant les années 1920 et publiée dans la Revue générale des chemins de fer en 1929 (fig. 4), on voit que les trois dépôts des machines jadis situés entre la rue de Tolbiac et le boulevard Masséna ont été démolis pour laisser la place à deux faisceaux de voies consacrées au matériel vide et aux messageries. Même le dépôt le plus récent a disparu, celui qui était situé entre la rue Watt et le boulevard Masséna, muni d'un pont tournant de 23,50 m et qui avait nécessité la mise en place d'un tablier supplémentaire sur le pont Watt en 1908. Ces nouveaux faisceaux ont nécessité l'allongement des ponts de la rue Watt côté Chevaleret et l'adjonction de tabliers supplémentaires ; mais on comprend au vu de ces schémas publiés en 1929 pourquoi la compagnie aurait préféré la suppression pure et simple de la rue Watt.

Les arguments présentés par M. Balling en 1917 semblent avoir retenu l'attention de sa hiérarchie. Cependant, déjà dès le premier lancement de son idée, on voit que la difficulté - voire l'impossibilité -

26- Les arrondissements sont les circonscriptions territoriales d'exploitation de la compagnie pour chaque service (exploitation, matériel et traction, voie et travaux) ; le $1^{\text {er }}$ recouvre la tête du réseau, donc pour Voie et Travaux les gares de Paris-Austerlitz, Chevaleret et Ivry. L'ingénieur en chef dirige l'arrondissement, l'ingénieur en chef de la Voie et des Travaux l'ensemble du service de la compagnie.

27- SNCF, CAH 40LM71/6/20/3 : Lettre de M. Balling, ingénieur en chef du $1^{\text {er }}$ arrondissement, à M. Couvrat-Desvergnes, ingénieur en chef de la Voie et des Travaux. 4 juillet 1917. Note manuscrite de M. Couvrat-Desvergnes à M. Bloch (ingénieur en chef de l'Exploitation), $1^{\text {er }}$ août 1917. 
d'obtenir l'autorisation de supprimer la rue Watt n'avait pas échappé à ses collègues. «Il serait possible de sonder la ville de Paris au sujet de la suppression de la rue Watt », écrit Couvrat-Desvergnes en août 1917 ; mais cela lui paraît néanmoins « un gros morceau à enlever $»^{28}$. La Compagnie d'Orléans se lance alors dans une stratégie ambiguë qu'elle maintiendra jusqu'en 1923 : tout en ayant peu d'espoir d'obtenir la suppression qui lui serait avantageuse, elle tente ses chances, insistant auprès de la ville et du ministère sur le fait que cette suppression lui est indispensable. À l'intérieur de la compagnie, d'autres solutions sont pourtant envisagées et débattues. M. Balling écrit en septembre 1917 qu'on « peut, en effet, prolonger la rue Regnault sous nos voies pour rejoindre la rue Croix-Jarry, mais le remède vaudrait le mal $»^{29}$. Il est persuadé que le « jour viendra où [...] nous devrons faire une cour dans l'espace entre la rue Watt, le quai et l'usine Popp ${ }^{30}$; opération qui serait rendue difficile par la solution que propose son collègue visant à prolonger la rue Regnault. « Je préférerais tâcher d'obtenir la suppression de la rue Watt. »

La compagnie avait procédé à un comptage de la circulation dans la rue, d'où il résultait qu'elle était surtout utilisée par des piétons (1 300 par jour environ) et moins par des voitures à cheval (300 environ). Pour la compagnie, une passerelle pour piétons suffisait pour remplacer donc la rue Watt; une appréciation qui diffère de celle des riverains comme de celle des ingénieurs de la ville.

Il est intéressant de voir comment dans ce contexte la compagnie tente d'agir sur l'aménagement urbain pour lui faire servir ses intérêts. «C'est une affaire d'argent, et si la ville y a intérêt, elle tâchera de faire aboutir l'affaire. Si d'ailleurs nous achetons les terrains voisins de la rue Watt, nous supprimerons par là même une partie du trafic de cette rue $^{31} »$. Deux ans plus tard, M. Balling insiste encore : «Il n'y a qu'une solution qui présente pour nous un avantage sérieux, c’est la suppression

\footnotetext{
28- Ibid.

29- SNCF, CAH 40LM71/6/20/2 : Copie de lettre de M. Balling, ingénieur du $1^{\text {er }}$ arrondissement, à $\mathrm{M}$. Semelin, Inspecteur principal de l'Exploitation. 3 septembre 1917.

30- Usine Popp ou usine de la SUDAC, Société urbaine d'air comprimé : notons qu’une partie de ses anciens bâtiments a été reconvertie pour être intégrée à l'École nationale supérieure d'architecture de Paris Val-de-Seine, dans le cadre de la transformation récente du quartier.
}

31- Voir doc. cit. note 29. 
pure et simple de la rue Watt. J'admets qu'elle a peu de chances d'aboutir ; on pourrait néanmoins tenter la chose, d'autant que nous pourrions en échange verser à la ville une indemnité importante ${ }^{32}$. »

\section{Un projet contesté par les riverains}

On voit que, en 1923, alors même que les riverains de la rue Watt adressent au ministre des Travaux publics une pétition pour protester contre le projet de suppression, la Compagnie d'Orléans élabore des projets en vue de maintenir la rue en la déviant ${ }^{33}$. La pétition adressée par les « habitants, industriels et commerçants du XIII ${ }^{\mathrm{e}}$ arrondissement » est en soi un document intéressant en tant que témoignage de l'activité ouvrière et commerciale dans cette partie de Paris pendant les années 1920 :

« [...] Le quartier de la gare n'est desservie [sic], entre le Pont de Tolbiac et le Pont National, que par la rue Watt [...et] son trafic ne fait qu'augmenter de jour en jour [...] Les débarquements qui s'opèrent entre le Pont de Tolbiac et le Pont National n'ont que la rue Watt, pour desservir tout ce quartier, composé de : Marchands de bois - de charbons - de Plâtre - bitume - Asphalte - des Entrepôts de Papiers, et d'importantes Usines qui reçoivent leurs marchandises du Port de la Gare par cette rue [...] L'usage de la rue Watt par des piétons est d'une utilité incontestable ; elle est la seule qui conduit directement du Quai de la Gare à la rue du Chevaleret et à la Gare d'Orléans-Ceinture par la rue du Loiret qui lui fait suite. Sa suppression priverait d'un accès direct au Chemin de fer de Ceinture les nombreux ouvriers travaillant à l'Entrepôt Général de la Société Fermière de l'État pour les eaux de Vichy, dans les Grands Moulins de Paris, les Magasins Généraux de Paris, les Grandes Papeteries de France. Dans les grands chantiers de Bois : Poupinel et Parent - Chatelet - Lamoureux, etc. $[\ldots]^{34}$. »

Cette pétition est secondée par les interventions de M. Poupinel, président honoraire de la chambre syndicale des bois à œuvrer, des bois de sciage et d'industrie, qui adresse lui aussi des courriers au ministre des Travaux publics qui les fait suivre au directeur de la Compagnie

32- SNCF, CAH 40LM71/6/20 : Lettre d'Eug. Hérard, pour le Chef de l'Exploitation, à $\mathrm{M}$. Couvrat-Desvergnes, Ingénieur en Chef de la Voie et des Travaux, 15 septembre 1917. Note tapuscrite de l'Ingénieur principal du $1^{\text {er }}$ arrondissement, 2 août 1919 ; note manuscrite de M. Balling, 6 août 1919.

33- SNCF, CAH 40LM71/6/17: Lettres de M. l'ingénieur en chef Jullien à M. Leboulleux, 24 et 26 juillet 1923.

34- SNCF, CAH 40LM71/6/13 : Pétition adressée par le Comité de Défense des Intérêts du Quartier de la Gare à Monsieur le Ministre des Travaux publics, 11 juin 1923. 
d'Orléans ${ }^{35}$. Dans ses courriers, M. Poupinel affirme que la compagnie « pourrait obtenir satisfaction par un pont élevé au-dessus des voies ». La compagnie répond à cette affirmation par des arguments d'ordre technique, évoquant en particulier les contraintes imposées par la multiplicité des cotes de niveau sur le site : "si on voulait conserver la rue Watt à la traversée des voies nouvelles il faudrait l'abaisser, et la rampe de raccord avec la rue du Chevaleret aurait une pente excessive »; « les voies du saut-de-mouton prévu pour rendre indépendants les mouvements des machines [...] coupent la rue Watt à un niveau tel que la suppression de cette rue s'impose ${ }^{36}$. »

\section{Des contraintes techniques, arguments de poids}

Nous verrons que l'organisation du plateau des voies des gares d'Austerlitz, de Chevaleret et d'Ivry allait en effet en se complexifiant pendant cette période, notamment par l'aménagement d'un souterrain qui devait passer au-dessous des ponts de Magenta et de Ceinture. Le chantier de construction de ce souterrain nécessitait l'établissement de « voies de travaux»; entre saut-de-mouton et voies souterraines, le site exigeait une gestion toujours plus fine de l'aménagement des cotes de niveau qui étaient déjà multiples avant l'intensification de l'activité ferroviaire qui a marqué cette période. La rue Watt elle-même en témoigne bien, avec la pente et la rampe de son profil entre la rue du Chevaleret et le quai de la Gare, avec les voies du raccordement Ceinture et du PO qui la traversaient à des niveaux différents, avec les aménagements mis en place en cas de crue de la Seine - trottoir surélevé et pompes - et avec l'égout situé juste au-dessous de sa chaussée (fig. 10).

Les ingénieurs de la ville de Paris continuent cependant de poursuivre l'idée de faire maintenir la rue Watt grâce à l'établissement d'un « pont supérieur» - un pont qui passe par dessus les voies ferrées. Les ingénieurs de la Compagnie du Paris-Orléans réagiront en plaçant la question des cotes de niveau au cœur de certains échanges où ils semblent vouloir démontrer l'impossibilité technique de toute autre solution que celle de la suppression de la rue ${ }^{37}$.

35- SNCF, CAH 40LM71/6/13/8 : Lettre de M. Paul Poupinel, Président honoraire de la Chambre Syndicale des Bois à Euvrer, des Bois de Sciage et d'Industrie, à M. le Ministre des Travaux publics, 29 juin 1923.

36- Ibid., Observations dactylographiées de l'ingénieur en chef du $1^{\text {er }}$ arrondissement sur la lettre de M. Poupinel.

37- SNCF, CAH 40LM71/6/17 : Lettres de M. l'ingénieur en chef Jullien à M. Leboulleux, 24 et 26 juillet 1923 . 


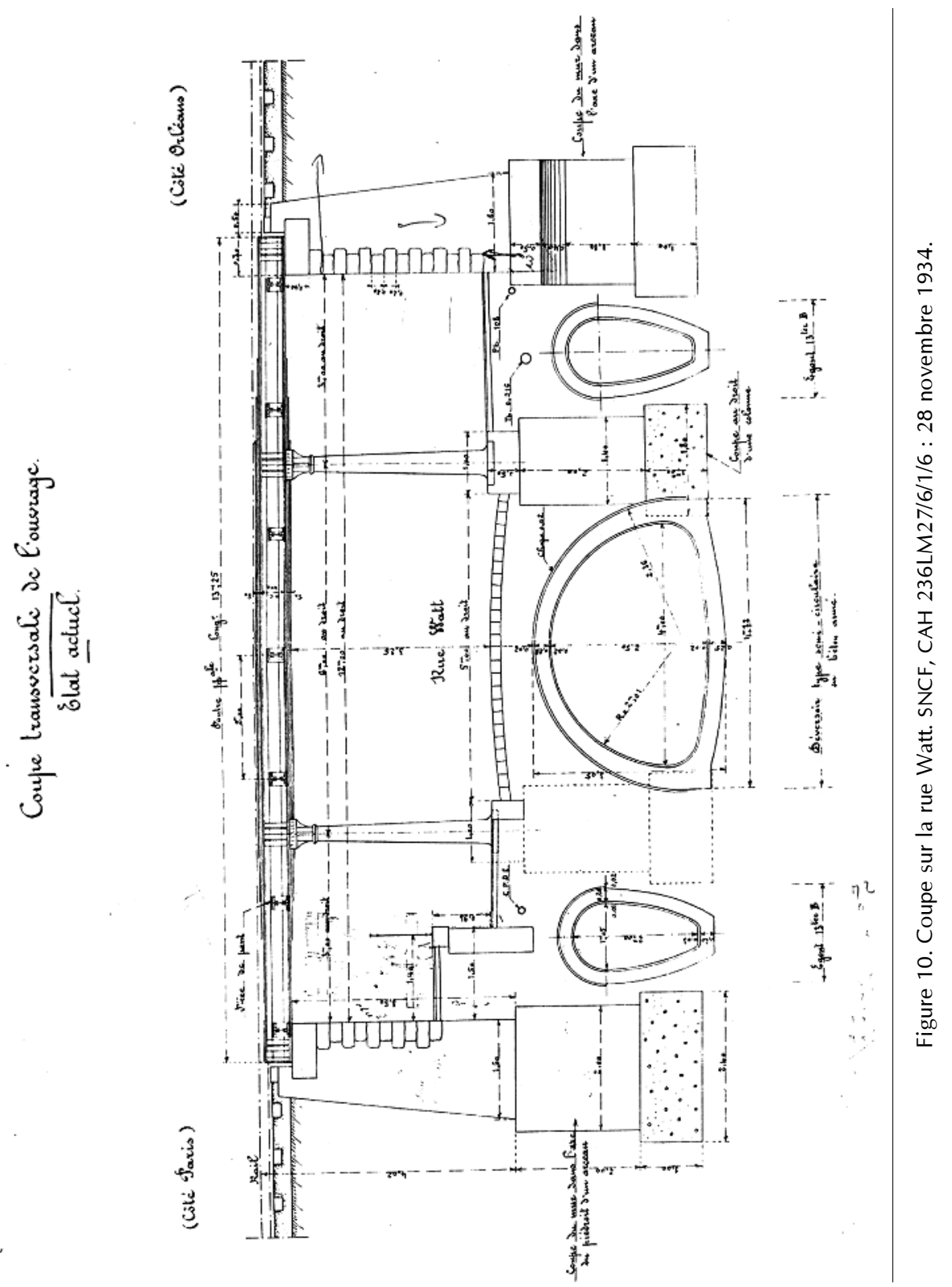




\section{La rue Watt maintenue}

Les discussions continuent pendant la fin de l'année 1923 et le début de l'année 1924 : il est intéressant de noter les termes dans lesquelles l'ingénieur de la ville de Paris présente l'importance, pour le quartier, du maintien de la rue Watt. Reprenant certains arguments des signataires de la pétition du 11 juin 1923, M. Biette, l'inspecteur général des services techniques de la voie publique, souligne que « le préjudice subi par les riverains » en cas de suppression de la rue Watt «serait d'autant plus sensible que par le fait même de l'existence du chemin de fer d'Orléans, le réseau des voies publiques de cette partie du XIII ${ }^{\mathrm{e}}$ arrondissement est très peu serré, et que pour trouver une rue parallèle à la rue Watt, il faut vers le nord, franchir 500 mètres pour gagner la rue de Tolbiac et vers le sud, 200 mètres pour atteindre le Boulevard Masséna [...] Ainsi, la suppression projetée d'un tronçon de la rue Watt désorganise le réseau des voies publiques du XIII arrondissement [...] $\gg^{38}$.

La Compagnie d'Orléans conteste cette dernière conclusion mais admet en interne que " nous éviterons difficilement le maintien de la rue Watt $»^{39}$. Dans une lettre à l'ingénieur du contrôle du réseau d'Orléans au ministère, la direction de la compagnie continue à maintenir que : « la rue Watt, n'aboutissant à aucun pont sur la Seine, ne sert qu'à une circulation essentiellement locale et très limitée entre les riverains et ceux des voies voisines, circulation qui serait assurée aussi facilement par le Boulevard Masséna que par le pont supérieur accolé à la Ceinture dont la construction nous est suggérée. » Cependant, la compagnie affirme maintenant qu'il serait " possible de conserver la rue Watt moyennant un abaissement local de $1 \mathrm{~m} 20 »$; une modification du tracé, le rendant plus sinueux, serait également nécessaire dans cette configuration ${ }^{40}$.

Cette proposition est acceptée par les ingénieurs de la ville de Paris, à la condition cependant de certains aménagements visant à préserver l'ouvrage de risques de crues. Ces mesures sont contestées dans le détail sinon dans leur principe par les ingénieurs de la Compagnie du Paris-Orléans, qui semblent vouloir insister sur la supériorité de leur

38- SNCF, CAH 40LM71/6/15: Préfecture de la Seine. Service Technique de la Voie Publique. Compagnie d'Orléans. Projet d'extension des Gares de Paris-Austerlitz et Paris-Ivry. Avis de l'inspecteur général, 25 septembre 1923.

39- SNCF, CAH 40LM71/6/15: Observations de l'ingénieur en chef du $1^{\text {er }}$ arrondissement sur l'Avis des Services Techniques de la Voie Publique, 15 octobre 1923.

40- SNCF, CAH 40LM71/6/14 : Lettre signée par M. Paul-Dubois pour le directeur de la Compagnie, à Monsieur Delacourcelle, Ingénieur en Chef du Contrôle de la Voie et des Bâtiments (Ministère des Travaux publics), 29 octobre 1923. 
compétence et de leur maitrise de ces questions techniques afférentes aux caractéristiques spécifiques de terrains situés à l'intérieur des emprises ferroviaires ${ }^{41}$.

En septembre 1927, le directeur de la compagnie peut enfin écrire au préfet de la Seine que "par mesure d'économie et pour obvier aux inconvénients signalés, il nous serait possible maintenant et pour longtemps encore de conserver à la rue son tracé rectiligne et de maintenir le point bas à son niveau actuel $»^{42}$. Après dix ans d'études et de discussions, la rue Watt restera presque telle qu'elle était en 1917, même si son profil devra être légèrement modifié du côté de la rue du Chevaleret pour s'accommoder de la mise en place de nouveaux tabliers.

Le projet finalement adopté est conçu pour être réalisé en deux phases, la première devant être exécutée en 1928, la deuxième destinée à n'être entreprise «que dans quelques années si les besoins du trafic nous y obligeaient ${ }^{43}$. Les modifications du profil de la rue seraient effectuées dès la première phase, même si elles ne devaient en principe être nécessaires que lors de la deuxième ; celle-ci devait couvrir par de nouveaux tabliers la partie de la rue Watt qui descendait depuis la rue du Chevaleret pour passer sous les voies ferrées. La rue a donc été abaissée et couverte par un tablier en poutrelles d'une seule portée, enrobées dans du béton, sur une longueur de 70 mètres pour permettre la pose de nouvelles voies à l'emplacement des anciens dépôts.

Une dernière modification des ponts est mentionnée dans notre documentation comme ayant été effectuée en 1937 : il s'agissait d'un remplacement de quatre tabliers sous voies principales non prévu dans les deux phases annoncées en 1927. Le schéma publié en 1929 (fig. 4) donne en effet l'impression que les deux phases avaient été mises en œuvre de suite et étaient déjà réalisées à cette date. Les textes établis au moment des vérifications et épreuves de l'ouvrage suggèrent plutôt que les modifications de 1937 participent à un renforcement nécessité par l'augmentation du poids des machines à l'essieu ${ }^{44}$.

41- SNCF, CAH 40LM71/6/13/3: Projet d'extension des gares de Paris-Austerlitz et Paris-Ivry. Avis complémentaire de l'Inspecteur Général des Ponts et Chaussées, Inspecteur Général des Services Techniques de la Voie Publique, 3 mars 1924 ; SNCF, CAH 40LM71/6/13/2: Observations de M. l'Ingénieur en Chef du $1^{\text {er }}$ arrondissement à M. l'Ingénieur en Chef (de la Voie et des Travaux), concernant l'avis de l'Inspecteur Général des Services Techniques de la Voie Publique du 3 mars 1924, 5 avril 1924. 42- SNCF, CAH 40LM71/6/10: Projet de lettre à Monsieur le Préfet de la Seine de H. Bréaud, Directeur Général de la Compagnie, 21 septembre 1927.

43- Ibid.

44- SNCF, CAH 236LM27/6/3 : Chemin de fer d’Orléans. Service de la voie. P.I. de la Rue Watt. $4^{\circ}$ Procès-verbaux des Visites détaillées, 13 mars 1903 ; 30 juin 1905 ; 6 mai 1910 ; 22 juin 1933. 


\section{Un accident symptomatique?}

Un accident survenu sur les ponts de la rue Watt en 1929 nous paraît révélateur de la complexité des aménagements que la Compagnie du Paris-Orléans mettait alors en œuvre sur le site ${ }^{45}$. La réalisation de l'agrandissement de la gare impliquait la construction, comme nous l'avons mentionné plus haut, d'une voie souterraine passant sous les voies de Ceinture et le boulevard Masséna. Avec l'achèvement du gros œuvre de ce souterrain, un train de matériaux était utilisé pour le travail de ballastage des futures voies de circulation des machines. Il empruntait une "voie de travaux» desservant le chantier de construction du souterrain par une rampe importante. « Par suite d'une trop forte poussée ou d'un élan mal calculé », le train a heurté les poutres principales d'un des tabliers de la rue Watt, les déplaçant sur deux mètres environ, ainsi que les colonnes en fontes intermédiaires qui les soutenaient.

Cet accident nous paraît révélateur d'une situation où la compagnie ne semble pas tout à fait en mesure de maîtriser l'échelle des modifications et des aménagements qu'elle avait elle-même mis en œuvre. À l'issue de notre étude, les logiques économiques ayant présidé aux stratégies adoptées par la compagnie pour les divers projets d'aménagement de la rue Watt ne paraissent pas tout à fait cohérentes. Elle n'a pas ménagé ses efforts pour économiser sur des éléments qui, avec le recul du temps, nous paraissent aujourd'hui relativement mineurs, alors que pour d'autres opérations elle semble avoir agi en pure perte. Elle a par exemple laissé en place le raccordement avec la Ceinture pendant une trentaine d'années, malgré les plaintes insistantes de la ville de Paris et même du ministère, pour éviter de prendre en charge des aménagements qui risquaient de «n'être utiles que peu de temps». Elle a passé six années à tenter d'obtenir la suppression de la rue Watt tout en sachant que cette solution n'avait «que peu de chances d'aboutir». Mais elle a néanmoins construit une rotonde équipée d'un pont tournant de $23,50 \mathrm{~m}$, l'un des plus grands du site, pour projeter de la démolir après moins de dix ans d'exploitation (1908-1917).

On ne peut qu'être impressionné, cependant, par l'énergie et la vigueur avec lesquelles les ingénieurs de la Compagnie d'Orléans menaient leurs projets. Nous avons vu combien ceux-ci mettaient en jeu une multiplicité d'acteurs et une grande complexité administrative dans un contexte d'évolutions radicales, tant techniques que d'exploitation.

45- SNCF, CAH 40LM71/6/4 : Rapport spécial sur l'accident survenu le 27 juin 1929 au pont métallique de la rue Watt, 11 juillet 1929. 
Les ponts de la rue Watt ne représentent qu'un élément relativement mineur à l'intérieur d'un vaste site ferroviaire et industriel. Mais l'histoire de leur construction donne un aperçu du fonctionnement d'une grande compagnie de chemins de fer lorsqu'elle aménage sa tête de réseau et gère ses relations avec la ville. 